\title{
In vitro suppression of Sclerotinia minor by a seaweed extract from Durvillaea potatorum and Ascophyllum nodosum
}

\author{
Scott W. Mattner • Oscar N. Villalta • Denise Wite • \\ Ian J. Porter • Tony Arioli
}

Received: 7 March 2014 / Accepted: 9 June 2014 / Published online: 19 June 2014

(C) Australasian Plant Pathology Society Inc. 2014

\begin{abstract}
Five in vitro bioassays demonstrated a capacity for an undiluted seaweed extract, made from a combination of Durvillaea potatorum and Ascophyllum nodosum, to suppress the growth of Sclerotinia minor by $18-100 \%$. Inundation in the seaweed extract significantly $(p \leq 0.05)$ suppressed growth of sclerotia by $90 \%$, and reduced disease severity in lettuce seedlings. A control buffered to the same $\mathrm{pH}(8.8)$ as the seaweed extract also significantly $(p \leq 0.05)$ suppressed the growth of sclerotia, but only by $22 \%$. This suggests that $\mathrm{pH}$ only partially explains the suppressive effect of the extract against $S$. minor, and undiscovered modes of action exist.
\end{abstract}

Keywords Kelp extract $\cdot$ Seasol ${ }^{\circledR} \cdot$ Lettuce $\cdot$ Bioassay

Sclerotinia minor is a devastating necrotrophic pathogen of a number of horticultural crops, including lettuce, green bean, cabbage, and broccoli (Villalta et al. 2012). It survives in soil for many years as melanised sclerotia, which infect crops following their germination. The fungus generally does not produce any secondary inoculum within a season, and therefore the density of viable sclerotia in soil and their pathogenicity are the most crucial factors influencing disease

S. W. Mattner $\cdot$ O. N. Villalta $\cdot$ D. Wite $\cdot$ I. J. Porter

Department of Environment and Primary Industries, Knoxfield,

VIC 3180, Australia

S. W. Mattner

Victorian Strawberry Industry Certification Authority, Toolangi,

VIC 3777, Australia

S. W. Mattner $(\bowtie) \cdot$ I. J. Porter

School of Botany, La Trobe University, Bundoora, VIC 3086,

Australia

e-mail: swmattner@hotmail.com

T. Arioli

Seasol International, Bayswater, VIC 3153, Australia epidemics (Wu et al. 2008). Currently, there are few fungicides or fumigants available for growers to control sclerotia and mycelia of $S$. minor in soil due to registration withdrawals (Villalta et al. 2012) and reduced efficacy with repeated use (Martin et al. 1990). Research has identified several alternatives that can reduce the viability of $S$. minor in soil, including biological controls (Villalta et al. 2012), biofumigation (Villalta et al. 2008), and raising soil pH (Wilson et al. 2005), but these are generally less effective and less reliable than chemical controls.

Since the inception of seaweed extracts as soil amendments in horticulture, authors have reported their potential benefits for crop health (Craigie 2010). Recent evidence has linked the effect of seaweed extracts on crop health to stimulation of resistance mechanisms in the host plant (e.g. Subramanian et al. 2011). Few published studies, however, have considered the direct role of seaweed extracts in reducing inoculum of fungal soil-borne pathogens (Kulik 1995). In laboratory bioassays, Ara et al. (2005) found that specific fractions from an extract of Spatoglossum asperum inhibited the growth of the soil-borne pathogens Macrophomina phaseolina, Rhizoctonia solani and Fusarium solani. They attributed the effect to various fatty acid esters identified in the fractions. Similarly, ether extracts from various species of brown, green and red algae showed fungicidal effects against two Aspergillus and Penicillium species grown in culture (Khaleafa et al. 1975). The authors postulated that the effect was due to the presence of halogenated phenolics in the extracts. Similarly, Chanthini et al. (2012) demonstrated that the direct antifungal activity of various species of red and green algae against the pathogen Alternaria solani correlated with their phenolic content.

We conducted four in vitro bioassays to test the hypothesis that a seaweed extract has the potential to directly suppress the growth of mycelia and sclerotia of S. minor (National Collection of Fungi, VPRI Herbarium, VPRI 41837, isolated from lettuce grown at Boneo, Victoria in 2008). This isolate was 
used in all experiments reported in this paper. Pre-testing with other isolates of $S$. minor from field-grown lettuce showed similar responses to treatments as the test isolate. The seaweed extract (Seasol Commercial ${ }^{\circledR}$, Seasol International, Bayswater, Victoria) was made from Durvillaea potatorum and Ascophyllum nodosum, using a commercialised alkaline hydrolysis process. Prior to use, the extract was sterilised by exposure to UV for $2 \mathrm{~h}$ in a laminar flow cabinet. Pre-testing showed that this method successfully sterilised small volumes $(15 \mathrm{ml})$ of the extract without affecting its bioactivity against $S$. minor. Distilled water formed the control and was also sterilised by exposure to UV. In all bioassays, agar plates containing the pathogen were incubated at $21^{\circ} \mathrm{C}$ in the dark. Colony diameters (average of two perpendicular planes for each colony) of $S$. minor were recorded after 2 and 5 days incubation as a measure of fungal growth.

1. Disk diffusion bioassay. Antimicrobial susceptibility disks (Oxoid, Basingstoke, UK) were individually soaked in $1 \mathrm{ml}$ aliquots of the treatments for $1 \mathrm{~h}$, and then allowed to dry in a laminar flow cabinet. Disks were placed in the centre of a Petri dish ( $84 \mathrm{~mm}$ diameter) containing potato dextrose agar (PDA, Oxoid). Three agar plugs $(8 \mathrm{~mm}$ diameter) containing mycelia were cut from the edge of an actively growing culture of $S$. minor and placed equidistant $(25 \mathrm{~mm})$ from the disk on the PDA plates.

2. Agar amendment bioassay. Treatment solutions (1 ml) were spread evenly over the surface of PDA plates, and allowed to absorb into the media for $2 \mathrm{~h}$ in a laminar flow cabinet. An agar plug containing mycelium of $S$. minor was then placed in the centre of each of the plates.

3. Mycelia inundation bioassay. Agar plugs containing mycelia of $S$. minor were submerged in $1 \mathrm{ml}$ of nutrient broth (Oxoid) in a Falcon tube. Treatment solutions (4 ml) were added to the tubes and mixed on a vortex shaker for $30 \mathrm{~s}$. The tubes were then placed in a rotating incubator for $16 \mathrm{~h}$. Agar plugs were retrieved from the tubes, rinsed in sterile distilled water, and briefly allowed to dry on sterile filter paper in a laminar flow cabinet. Individual plugs were then placed in the centre of a fresh PDA plate.

4. Sclerotia inundation bioassay $(a)$. Laboratory-grown sclerotia were harvested from the edges of cultures of $S$. minor on PDA plates. Sclerotia were submerged in Falcon tubes containing $4 \mathrm{ml}$ of the treatment solutions and $1 \mathrm{ml}$ of nutrient broth, and placed in a rotating incubator for $16 \mathrm{~h}$. Following this, sclerotia were rinsed in sterile distilled water and allowed to dry on sterile filter paper in a laminar flow cabinet, before transferring individually onto separate PDA plates (60 mm diameter).

Individual bioassays and the pathogenicity experiment (see below) were conducted as randomised complete block designs, with three blocks containing three replicate plates of each treatment. Where variation occurred across treatments, data were analysed by ANOVA using Genstat v. 12.1 (VSN International, Hemel Hempstead, UK), and Fisher's least significant difference test used to identify differences between treatment means (where $p \leq 0.05$ ). Homogeneity of variance was determined by examining plots of fitted values versus residuals, while histograms of residuals were examined for normality of distribution. Standard errors were calculated where variation occurred in one treatment only. Where no variation occurred in any treatment, there was no analysis of data.

At 2 days incubation, results from all bioassays demonstrated a capacity for the seaweed extract to significantly $(p \leq$ 0.05 ) suppress the growth of mycelia and sclerotia of $S$. minor compared with the control, with the effect ranging from 18$100 \%$ (Table 1). Overall, bioassays that involved inundation of $S$. minor in the seaweed extract (i.e. bioassays 3 and 4) showed greater inhibitory effects against fungal growth than bioassays conducted on agar surfaces (i.e. bioassays 1 and 2). This concurs with Hadacek and Greger (2000) who reported that inundating test pathogens in treatment solutions exposes them to a greater concentration of potential inhibitory compounds than bioassays conducted on agar surfaces.

By 5 days incubation, inundation of sclerotia in the seaweed extract had significantly $(p \leq 0.05)$ suppressed the ensuing growth of mycelia by $50 \%$ compared with the control (bioassay 4, Table 1). In contrast, inundation of mycelia in the seaweed extract killed the fungus, which was confirmed by sub-culturing on two separate occasions (bioassay 3, Table 1). These results are consistent with mycelia being more susceptible to inhibitory compounds and environmental factors than sclerotia, which have protective melanised rinds (Chet and Henis 1975). In the disk diffusion and agar amendment bioassays (bioassays 1 and 2, respectively), mycelia of the fungus had grown to the edge of the plate by 5 days of incubation, so the effect of treatment could no longer be determined (Table 1).

5. Sclerotia inundation bioassay (b). The alkaline hydrolysis process used to generate the seaweed extract gives the product a high $\mathrm{pH}(8-9)$. Optimal growth of Sclerotinia spp. in culture occurs at a low pH of 3-6 (Willetts and Wong 1980), and $\mathrm{pH}$ of $>8.0$ can suppress germination of their sclerotia (Wilson et al. 2005). For this reason we conducted a further sclerotia inundation bioassay, as described previously (bioassay 4), to test the hypothesis that the high $\mathrm{pH}$ of the seaweed extract suppresses the growth of $S$. minor. Treatments included: (a) undiluted extract from $D$. potatorum and $A$. nodosum, (b) water buffered with $1 \mathrm{M}$ sodium borate to the same $\mathrm{pH}(8.8)$ as the seaweed extract, (c) a 1:500 dilution of seaweed extract, which is the lowest application concentration in the field and had an equivalent $\mathrm{pH}(7.5)$ to the water control, (d) 
Table 1 Colony diameters (mm) of Sclerotinia minor treated with a commercial extract from Durvillaea potatorum and Ascophyllum nodosum in various in vitro bioassays. LSD is the least significant difference where $p=0.05$. Values in square parentheses are standard errors

\begin{tabular}{|c|c|c|c|c|c|c|}
\hline \multirow[t]{2}{*}{ Bioassay method } & \multicolumn{3}{|l|}{2 Days incubation } & \multicolumn{3}{|l|}{5 Days incubation } \\
\hline & Seaweed extract & Control (water) & $\operatorname{LSD}(p=0.05)$ & Seaweed extract & Control (water) & $\operatorname{LSD}(p=0.05)$ \\
\hline 1. Disk diffusion & 32.9 & 44.1 & 4.7 & $60.0^{\dagger}$ & $60.0^{\dagger}$ & - \\
\hline 2. Agar Amendment & 37.7 & 46.0 & 3.2 & $84.0^{\dagger}$ & $84.0^{\dagger}$ & - \\
\hline 3. Mycelia inundation & 0.0 & $44.0[0.9]$ & - & 0.0 & $84.0^{\dagger}$ & - \\
\hline 4. Sclerotia inundation & 0.0 & $8.7[0.5]$ & - & $31.1[0.9]$ & $60.0^{\dagger}$ & - \\
\hline
\end{tabular}

$\dagger$ Colonies had reached the edge of the agar plate

distilled water as a control, (e) freshly harvested sclerotia from actively growing cultures of $S$. minor as a laboratory control, and (f) allyl isothiocyanate (Sigma-Aldrich, St Louis, USA), which is a proven biofumigant treatment for control of sclerotia (Villalta et al. 2008). Colony growth (diameter) of S. minor was measured after 2, 3, 4 , and 5 days incubation.

Inundation of sclerotia in undiluted seaweed extract significantly $(p \leq 0.05)$ delayed their germination by 3 days and reduced the ensuing growth of mycelia by $90 \%$ (at 5 days of incubation), compared with the water control (Table 2). By comparison, water buffered to the same $\mathrm{pH}$ as undiluted seaweed extract also significantly $(p \leq 0.05)$ suppressed the growth of mycelia from germinated sclerotia, but only by $22 \%$ (significantly, $p \leq 0.05$, less than the effect of the undiluted seaweed extract). Diluting the seaweed extract to a low concentration significantly $(p \leq 0.05)$ suppressed the growth of mycelia from germinated sclerotia by $30 \%$ compared with the water control (which was at an equivalent $\mathrm{pH}$ ). There was no difference $(p>0.05)$ in the growth of mycelia from germinated sclerotia in the water and laboratory controls, but allyl isothiocyanate killed sclerotia.

Table 2 Colony diameters $(\mathrm{mm})$ of Sclerotinia minor treated with a commercial extract from Durvillaea potatorum and Ascophyllum nodosum and various controls, in a sclerotia inundation bioassay (bioassay 5). LSD is the least significant difference where $p=0.05$

\begin{tabular}{lllll}
\hline Treatment & \multicolumn{4}{l}{ Incubation period } \\
\cline { 2 - 5 } & 2 days & 3 days & 4 days & 5 days \\
\hline $\begin{array}{l}\text { Seaweed extract (undiluted), } \\
\text { pH 8.8 }\end{array}$ & 0.0 & 0.0 & 0.0 & 5.3 \\
Buffered water, pH 8.8 & 0.0 & 4.0 & 15.8 & 41.2 \\
Seaweed extract (1:500), pH 7.5 & 0.0 & 4.7 & 16.7 & 37.7 \\
Water (negative control), pH 7.4 & 4.9 & 11.1 & 24.0 & 53.2 \\
Laboratory control & 5.4 & 12.7 & 26.6 & 53.3 \\
Allyl isothiocyanate (positive & 0.0 & 0.0 & 0.0 & 0.0 \\
$\quad$ control), pH ND & - & 3.2 & 4.2 & 6.4 \\
LSD ( $p=0.05)$ & & &
\end{tabular}

This bioassay demonstrated that the high $\mathrm{pH}$ of undiluted seaweed extract only partially explains its suppressive effect against $S$. minor. Furthermore, there was no evidence that $\mathrm{pH}$ contributed to the action of the extract when it was diluted to the lowest concentrations applied in the field. Previous research has attributed the direct suppressive effects of extracts from other species of marine algae to their content of fatty acid esters (Ara et al. 2005), phenolics (Khaleafa et al. 1975, Chanthini et al. 2012), and betaines (Wu et al., 1998). Koivistoinen et al. (1959) found that indole acetic acid, which is one of several plant growth regulators contained in the seaweed extract used in the current experiments (Seasol International 2013), inhibits the growth of Sclerotinia trifoliorum in culture. This suggests that the mechanism of extract from $D$. potatorum and A. nodosum in suppressing $S$. minor may involve a complex mixture of organic compounds, in addition to high $\mathrm{pH}$. Further research is proposed to understand the mode of suppressive action, and to fractionate and characterise the active components of the seaweed extract against $S$. minor, and other soil-borne pathogens.

We conducted an in vitro pathogenicity experiment using the method described by Christensen et al. (1988) to test the hypothesis that treatment of sclerotia of $S$. minor with the seaweed extract reduces its ability to cause disease in a host. One hundred seeds of lettuce var. Great Lakes (New Gippsland Seeds and Bulbs, Silvan, Victoria) were surface

Table 3 Disease score ${ }^{\dagger}$ of lettuce seedlings infected with Sclerotinia minor treated with commercial extracts from Durvillaea potatorum and Ascophyllum nodosum. LSD is the least significant difference where $p=$ 0.05

\begin{tabular}{ll}
\hline Treatment & Disease score $^{\dagger}(0-3)$ \\
\hline Seaweed extract (undiluted) & 0.97 \\
Seaweed extract (1:500) & 1.53 \\
Water (negative control) & 2.58 \\
Laboratory control & 2.56 \\
LSD ( $p=0.05$ ) & 0.31 \\
\hline${ }^{\dagger} 0=$ healthy green shoots, $1=<50 \%$ of shoot system wilted, $2=>50 \%$ \\
of shoot system wilted, $3=$ shoots rotted
\end{tabular}


sterilised for $3 \mathrm{~min}$ in a $70 \%$ ethanol $/ 10 \%$ sodium hypochlorite solution, followed by three rinses in sterile distilled water, and dried in a laminar flow cabinet on sterile filter paper. Seed was then placed in a Falcon tube and soaked in sterile distilled water overnight $(12 \mathrm{~h})$ at $21^{\circ} \mathrm{C}$. Four seeds were placed in a line (20 mm apart) across a water agar (Sigma-Aldrich) plate (84 $\mathrm{mm}$ diameter). After 3 days germination, when roots had extended half way across the plate, a treated sclerotium of $S$. minor was placed $10 \mathrm{~mm}$ from each of the seedlings. Sclerotia were treated as described previously with: (a) undiluted extract from $D$. potatorum and A. nodosum, (b) a 1:500 dilution of the seaweed extract, (c) distilled water as a control, or (d) were freshly harvested from actively growing cultures as a laboratory control. Plates were incubated at $21^{\circ} \mathrm{C}$ in the light for a further 7 days, and the severity of shoot disease scored using the scale described in Table 3.

Inundating sclerotia in undiluted seaweed extract significantly $(p \leq 0.05)$ reduced the severity of disease they caused in lettuce seedlings (Table 3). S. minor produces a range of pectolytic and cellulolytic enzymes, and oxalic acid that degrade host tissue. Production of these enzymes is optimal under low $\mathrm{pH}$ (5.0), and acidic conditions enhance the aggressiveness of the pathogen (Willetts and Wong 1980). For example, Wilson et al. (2005) found that raising soil $\mathrm{pH}$ with calcium hydroxide reduced disease incidence caused by S. minor in lettuce by $58 \%$. Therefore it is possible that the reduction in disease severity caused by the undiluted seaweed extract in the current experiment is due to the high $\mathrm{pH}$ of the product, even though its $\mathrm{pH}$ only played a partial role in the direct suppression of the pathogen (see previously). Further controlled trials, however, are needed to confirm this. Other researchers have demonstrated that extracts from $A$. nodosum can stimulate host resistance against Sclerotinia pathogens (Subramanian et al. 2011), and this may also explain the ability of the extract to reduce disease severity in the current experiment. Inundation of sclerotia in seaweed extract diluted to the lowest concentrations applied in the field also significantly $(p \leq 0.05)$ reduced disease severity in lettuce seedlings, but not to the same degree as undiluted extract (Table 3). This suggests that dilutions of the seaweed extract used in the field to maximise crop growth may differ from those needed to maximise suppression of disease caused by $S$. minor, and potentially other soil-borne pathogens.

It is important to note that sclerotia used in the current experiments were laboratory-grown and potentially less tolerant of inhibitory compounds and environmental factors than field-hardened sclerotia. Also concentrations of the seaweed extract used in the experiments were generally higher and exposure of $S$. minor to the extract longer and more controlled than what would normally occur in the field. This was done to determine the maximum potential for the seaweed extract to suppress $S$. minor. Consequently, further pot and field trials are needed to expand the results from the current bioassays to farm applications. Nonetheless, previous studies have shown that other commercial extracts from $A$. nodosum have the capacity to reduce disease incidence caused by Sclerotinia spp. in the field and glasshouse (Zhang et al. 2003; Guinan et al. 2013). This combined with a potential of extracts from $D$. potatorum and A. nodosum to directly suppress the growth and pathogenicity of $S$. minor, shows that further field research is warranted.

We conclude that a seaweed extract from $D$. potatorum and $A$. nodosum has a potential to directly suppress the growth of S. minor, and reduce its ability to cause disease. This effect is only partially due to the alkaline $\mathrm{pH}$ of the undiluted extract, and other organic compounds in the extract are likely to be involved.

\section{References}

Ara J, Sultana V, Qasim R, Ehteshamul-Haque S, Ahmad VU (2005) Biological activity of Spatoglossum asperum: a brown alga. Phytother Res 19:618-623

Chanthini K, Kumar CS, Kingsley SJ (2012) Antifungal activity of seaweed extracts against phytopathogen Alternaria solani. J Acad Indus Res 1:86-90

Chet I, Henis Y (1975) Sclerotial morphogenesis in fungi. Annu Rev Phytopathol 13:169-192

Christensen MJ, Falloon RE, Skipp RA (1988) A Petri plate technique for testing pathogenicity of fungi to seedlings and inducing fungal sporulation. Aust Plant Path 17:45-47

Craigie JS (2010) Seaweed extract stimuli in plant science and agriculture. J Appl Phycol 23:371-393

Guinan KJ, Sujeeth N, Copeland RB, Jones PW, O'Brien NM, Sharma HSS, Prouteau PFJ, O'Sullivan JT (2013) Discrete roles for extracts of Ascophyllum nodosum in enhancing plant growth and tolerance to abiotic and biotic stress. Acta Hort 1009:127-135

Hadacek F, Greger H (2000) Testing of antifungal natural products: methodologies, comparability of results and assay choice. Phytochem Anal 11:137-147

Khaleafa AF, Kharboush MAM, Metwalli A, Moshen AF, Serwi A (1975) Antibiotic (fungicidal) action from extracts of some seaweeds. Bot Mar 18:163-165

Koivistoinen P, Risser E, Poiijakallio O (1959) The inhibitory effect of certain indole compounds upon the growth of Sclerotinia trifoliorum Erikss. Acta Agric Scand 9:403-411

Kulik MM (1995) The potential for using cyanobacteria (blue green algae) and algae in the biological control of plant pathogenic bacteria and fungi. Eur J Plant Pathol 101:585-599

Martin C, Vega D, Bastide J, Davet P (1990) Enhanced degradation of iprodione in soil after repeated treatments for controlling Sclerotinia minor. Plant Soil 127:140-142

Seasol International (2013) Analysis of Seasol commercial concentrate. Seasol International, Bayswater, Victoria. URL: http://www. commercial.seasol.com.au/

Subramanian S, Sangha JS, Gray BA, Singh RP, Hiltz D, Critchley AT, Prithiviraj B (2011) Extracts of the marine brown macroalga, Ascophyllum nodosum, induce jasmonic acid dependent systemic resistance in Arabidopsis thaliana against Pseudomonas syringae pv. tomato DC3000 and Sclerotinia sclerotiorum. Eur J Plant Path 131:237-248 
Villalta ON, Wite D, Hunt J, Stewart A, Porter IJ (2012) Biological control of Sclerotinia minor on lettuce using Trichoderma and Coniothyrium species. Acta Hort 944:51-58

Villalta ON, Wite D, Porter IJ, Ames A, Imsic M (2008) Evaluating biofumigation amendments for the management of diseases caused by sclerotial pathogens in vegetable crops. In: Kirkegaard JA (ed) Proceedings of the Third International Biofumigation Symposium. CSIRO, Canberra, $\mathrm{p} 66$

Willetts HJ, Wong JAL (1980) The biology of Sclerotinia sclerotiorum, S. trifoliorum and $\mathrm{S}$. minor with emphasis on specific nomenclature. Bot Rev 46:101-165

Wilson CR, deLittle JA, Wong JAL, Schupp PJ, Gibson LJ (2005) Adjustment of soil-surface $\mathrm{pH}$ and comparison with conventional fungicide treatments for control of lettuce drop (Sclerotinia minor). Plant Pathol 54:393-400

Wu BM, Subbarao KV, Liu YB (2008) Comparative survival of sclerotia of Sclerotinia minor and S. sclerotiorum. Phytopath 98:659-665

Wu Y, Jenkins T, Blunden G, von Mende N, Hankins SD (1998) Suppression of fecundity of the root-knot nematode, Meloidogyne javanica, in monoxenic cultures of Arabidopsis thaliana treated with an alkaline extract of Ascophyllum nodosum. J App Phycol 10:91-94

Zhang X, Ervin EH, Schmidt RE (2003) Physiological effects of liquid applications of a seaweed extract and humic acid on creeping bentgrass. J Amer Soc Hort Sci 128:492-496 